\title{
The effects of item familiarity on the neural correlates of successful associative memory encoding
}

\author{
Nancy A. Dennis ${ }^{1}$ - Indira C. Turney ${ }^{1}$ - Christina E. Webb ${ }^{1}$ - Amy A. Overman ${ }^{2}$
}

Published online: 5 May 2015

(C) Psychonomic Society, Inc. 2015

\begin{abstract}
Associative memory is considered to be resource-demanding, requiring individuals to learn individual items and the specific relationships between those items. Previous research has shown that prior studying of items aids in associative memory for pairs composed of those same items, as compared to pairs of items that have not been prelearned (e.g., Kilb \& NavehBenjamin, 2011). In the present study, we sought to elucidate the neural correlates mediating this memory facilitation. After being trained on individual items, participants were scanned while encoding item pairs composed of items from the pretrained phase (familiarized-item pairs) and pairs whose items had not been previously learned (unfamiliarized-item pairs). Consistent with previous findings, the overall subsequent recollection showed the engagement of bilateral parahippocampal gyrus (PHG) and hippocampus, when compared to subsequent forgetting. However, a direct comparison between familiarizedand unfamiliarized-item pairs showed that subsequently recollected familiarized-item pairs were associated with decreased activity across much of the encoding network, including bilateral PHG, hippocampus, prefrontal cortex, and regions associated with item-specific processing within occipital cortex. Increased activity for familiarized-item pairs was found in a more limited set of regions, including bilateral parietal cortex, which has been associated with the formation of novel associations. Additionally, activity in the right parietal cortex correlated with associative memory success in the familiarized condition. Taken
\end{abstract}

Nancy A. Dennis

nad12@psu.edu

1 Department of Psychology, Pennsylvania State University, 450 Moore Building, University Park, PA 16802, USA

2 Department of Psychology and Neuroscience Program, Elon University, Elon, NC, USA together, these results suggest that prior exposure to items can reduce the demands incurred on neural processing throughout the associative encoding network and can enhance associative memory performance by focusing resources within regions supporting the formation of associative links.

Keywords Associative memory $\cdot$ Encoding $\cdot$ Parietal cortex · fMRI

A substantial amount of research has shown that associative memory, or memory for the relations between two or more items, is a more difficult memory task than item memory (Castel \& Craik, 2003; Gold, Hopkins, \& Squire, 2006; Kilb \& Naveh-Benjamin, 2007; Naveh-Benjamin, 2000; Overman \& Becker, 2009; Yonelinas, 1997; Yonelinas et al., 2007). This finding has been attributed to several factors, including differences in the retrieval mechanisms underlying item and associative information (i.e., item memory is supported by both recollection and familiarity, whereas associative memory relies primarily on recollection; Hockley \& Consoli, 1999; Yonelinas et al., 2007), interference associated with preexisting associations (Dolan \& Fletcher, 1997; Graf \& Schacter, 1987), and increased workload imposed by the need to simultaneously learn both item and associative information (Chalfonte \& Johnson, 1996; Naveh-Benjamin, 2000). Supporting the latter explanation, neuroimaging studies have shown that both item and associative encoding utilize many of the same neural regions, including the prefrontal cortex (PFC), parietal cortex, and sensory processing regions (e.g., visual cortex; Dennis et al., 2008; Giovanello \& Schacter, 2012; Giovanello, Schnyer, \& Verfaellie, 2004; Slotnick, Moo, Segal, \& Hart, 2003; Yonelinas, Hopfinger, Buonocore, Kroll, \& Baynes, 2001). Consequently, when both items and their associations need to be learned simultaneously, the 
encoding network may be taxed because it needs to divide resources between the two tasks. Such a division of neural resources may, in turn, lead to poorer performance in associative encoding than in a condition in which only associative information is being encoded.

In an effort to examine ways to reduce the demands imposed by associative memory tasks, studies have employed both item and pair repetition during encoding (Earles \& Kersten, 2008; Kilb \& Naveh-Benjamin, 2011; Light, Patterson, Chung, \& Healy, 2004; Overman \& Becker, 2009; Overman \& Stephens, 2013). Although pair repetition trains individuals on the specific item-item relationship through repeated exposure to the pairs, item repetition provides added exposure only to the individual items, thus eliminating the need to learn the items during later associative encoding. Although past studies have primarily used item repetition as a control for examining the effects of pair repetition (Earles \& Kersten, 2008; Kilb \& Naveh-Benjamin, 2011), item repetition has been shown to exhibit significant advantages in associative memory, as compared with a study condition composed only of associative pairs rather than items (Earles \& Kersten, 2008; Kilb \& Naveh-Benjamin, 2011). Specifically, by exposing individuals to items prior to the associative study phase, research has demonstrated significant increases in the associative hit rate relative to when associative pairs were displayed for only a single presentation (Earles \& Kersten, 2008; Kilb \& Naveh-Benjamin, 2011). This result suggests that associative encoding is improved by reducing the demand on cognitive resources incurred by simultaneously encoding both item and associative information.

Accordingly, in the present study we sought to extend previous behavioral work by examining the neural encoding activity related to subsequent associative recollection, as a function of whether or not the items included in the associative encoding task had previously been learned. This allowed us to determine the extent to which item familiarization (i.e., preexposure to items that will later be presented in pairs) reduces the cognitive workload during associative encoding. Prior to the encoding of the associative pairs, we familiarized participants with half of the items that would be used in the associative memory task. We then compared the associative encoding for familiarized (i.e., prestudied) item-item pairs to that for unfamiliarized (i.e., those with no prior exposure) itemitem pairs. We posited that prior item familiarization would result in reduced neural activation within regions that supported both item and associative encoding and also would increase processing in regions that mediated associative binding.

Previous studies investigating the role of repetition in neural processing have found repetition suppression effects throughout the encoding network, including ventral visual regions, PFC, and the medial temporal lobe (MTL) (Desimone, 1996; Gonsalves, Kahn, Curran, Norman, \& Wagner, 2005; Kremers et al., 2014; Rand-Giovannetti et al., 2006; Vannini,
Hedden, Sullivan, \& Sperling, 2013). Reduced activity across these regions has been linked to more efficient cognitive processing and has been posited to reflect a "sharpening" of cortical representations (Desimone, 1996; Wiggs \& Martin, 1998). Thus, we expected to observe reduced activity in the familiarized-item pair condition, relative to that in the typical associative encoding task (i.e., unfamiliarized-item pair condition), within the PFC as well as within areas of the occipital cortex that are involved in the encoding of visual details - in particular, within the fusiform face area and the parahippocampal place area, which have been shown to process faces and places, respectively (Brewer, Zhao, Desmond, Glover, \& Gabrieli, 1998; Kanwisher, McDermott, \& Chun, 1997; Prince, Dennis, \& Cabeza, 2009; Puce, Allison, Gore, \& McCarthy, 1995; Rossion, Schiltz, \& Crommelinck, 2003).

With respect to the MTL, previous studies have shown repetition suppression effects only when the exact item or association was repeated across encoding trials (Gonsalves et al., 2005; Kremers et al., 2014; Vannini et al., 2013). The present study differs in that the familiarized-item pair condition includes items that have previously been encountered, while the association presented during encoding is novel. As such, it remains an open question whether the MTL, and specifically the hippocampus, would exhibit repetition suppression effects. Given the role of the MTL, and in particular the perirhinal and entorhinal cortices in item encoding (Davachi, 2006; Diana, Yonelinas, \& Ranganath, 2007; Ranganath, 2010a, 2010b; Staresina \& Davachi, 2008), we predict that the familiarized-item pair condition will result in reduced activity in this region during associative encoding, reflecting a reduced need to process or encode the individual items. However, given the role of the hippocampus proper in supporting associative binding in particular (Diana et al., 2007; Ranganath, 2010b), and the fact that the associative binding requirements are equivalent in both conditions, we predicted that item familiarization would not influence hippocampal activity in the present task.

In contrast to the aforementioned repetition suppression effects found in ventral visual cortex and the MTL, the parietal cortex has shown repetition enhancement effects during encoding (Kremers et al., 2014; Vannini et al., 2013). Moreover, these effects are found to be largest for associative memory success (as compared to item memory or memory failures; Kremers et al., 2014), suggesting that they reflect the formation of novel associations (Fuster \& Bressler, 2012; Gruber \& Muller, 2002, 2005; Kremers et al., 2014; Vannini et al., 2013). Thus, we also propose that in addition to reducing the demand on the brain regions that support both item and associative encoding, item familiarization would enhance associative encoding and subsequent memory processes by increasing neural activity within regions that support representations of novel associations (e.g., parietal cortex). 


\section{Method}

\section{Participants}

A total of 22 participants were recruited from the Pennsylvania State University community and were paid for their participation. Participants were screened for histories of neurological and psychiatric illness, learning disabilities, and drug/substance abuse. All participants provided written informed consent, and all procedures were approved by the Pennsylvania State University Institutional Review Board. Two participants were excluded from the present analyses due to failure to complete the task (one) and failure to follow the task instructions (one). The present analysis included 20 right-handed participants ( 12 female, eight male) between the ages of 18 and 29 years old (mean age $=23$ years, $S D=3.07$ ).

\section{Stimuli}

The stimuli consisted of 220 color photographs of faces $(50 \%$ male, $50 \%$ female) and 220 color photographs of scenes. The face stimuli consisted of both male and females faces, each exhibiting a neutral expression, taken from the following online databases: the Color Facial Recognition Technology (FERET) database (Phillips, Moon, Rizvi, \& Rauss, 2000; Phillips, Wechsler, Huang, \& Rauss, 1998), the adult face database from Denise Park's lab (Minear \& Park, 2004), the AR face database (Martinez \& Benavente, 1998), and the FRI Computer Vision Laboratory Face Database (Solina, Peer, Batageli, Juvan, \& Kovac, 2003). The scene stimuli consisted of outdoor and indoor scenes collected from an Internet image search. Using Adobe Photoshop CS2, version 9.0.2, and Irfanview 4.0 (www. irfanview.com), we edited the face stimuli to a uniform size $(320 \times 240$ pixels $)$ and background (black), and the scene stimuli were standardized to $576 \times 432$ pixels.

During the item familiarization phase (prior to the associative memory task), 90 faces and 90 scenes were presented separately to each participant. Item retrieval was tested following the initial training with the retrieval task consisting of the original 90 faces and scenes and 40 new faces and scenes (see the Results section). The associative encoding task incorporated 195 face-scene pairs (90 pairs of familiarized items and 105 pairs of unfamiliarized items). ${ }^{1}$ All pairs were presented against a black background with the face positioned to the left of center and the scene to the right (see Fig. 1). Retrieval consisted of 220 total trials: 130 encoding pairs (60 familiar and 70 unfamiliar pairs) were presented as targets (exact facescene pairings), and the remaining pairs were randomly

\footnotetext{
${ }^{1}$ Due to a programming error, the relational encoding included 15 additional unfamiliar pairs (three per run), which resulted in five additional unfamiliar lures and ten additional unfamiliar targets at retrieval, as compared to the familiarized condition.
}

recombined as related lures (new face-scene pairings: 30 familiarized, 35 unfamiliarized). Additionally, 25 completely novel face-scene combinations were also presented during retrieval, serving as novel lures.

\section{Procedure}

Item familiarization was completed outside of the scanner, prior to scanning. During this item-encoding phase, each image was presented for $2,500 \mathrm{~ms}$, during which time participants were asked to rate the friendliness of the faces and the pleasantness of the scenes (the faces and scenes were studied in alternating blocks). In order to verify that items were indeed learned, item memory was assessed using a yes/no recognition task in which each image was presented for 2,500 ms. Again, face and scene memory were tested in separate blocks. (Pilot testing showed that going through this process only once resulted in $75 \%$ accuracy across all participants; see the Results for the study-specific hit rates.)

Associative encoding and retrieval were carried out in the fMRI environment. During both associative encoding and retrieval, participants lay supine in the scanner while images were projected onto a screen that was viewed through a mirror mounted on the head coil. Behavioral responses were made with the right hand and were recorded using a four-key standard button box controller. The associative encoding consisted of five 4-min runs. Although all of the encoding pairs were novel, half of the pairs consisted of faces and scenes that had previously been studied by the participants (familiarized items) in the item-encoding phase. The other half of the pairs consisted of faces and scenes that were completely novel to the participants (unfamiliarized items; see Fig. 1). The presentation of each pair type was random across all runs. During encoding, each face-scene combination was presented for 3 , $000 \mathrm{~ms}$, during which time participants were asked to rate on a scale of 1-4 how well the face fit with the scene (i.e., how likely it was that the person would live, work, or vacation in the pictured scene). Participants were also informed that a memory test would follow.

During retrieval, the targets, recombined lures (created by recombining items of the encoded pairs), and novel lures (new pairs composed of faces and scenes never previously presented) were randomly intermixed and displayed for 4,000 ms, during which time participants made memory responses using the remember/know/new paradigm. The participants were specifically alerted to the inclusion of the three different trial types, and they were further instructed to respond "remember" if they were certain that the exact pairing had been presented in the previous task and if they could remember specific details about the association and the pairing's presentation from the study phase. In addition, participants were instructed to respond "know" if the exact face-scene pair looked familiar but their memory was lacking any specific details of its prior 


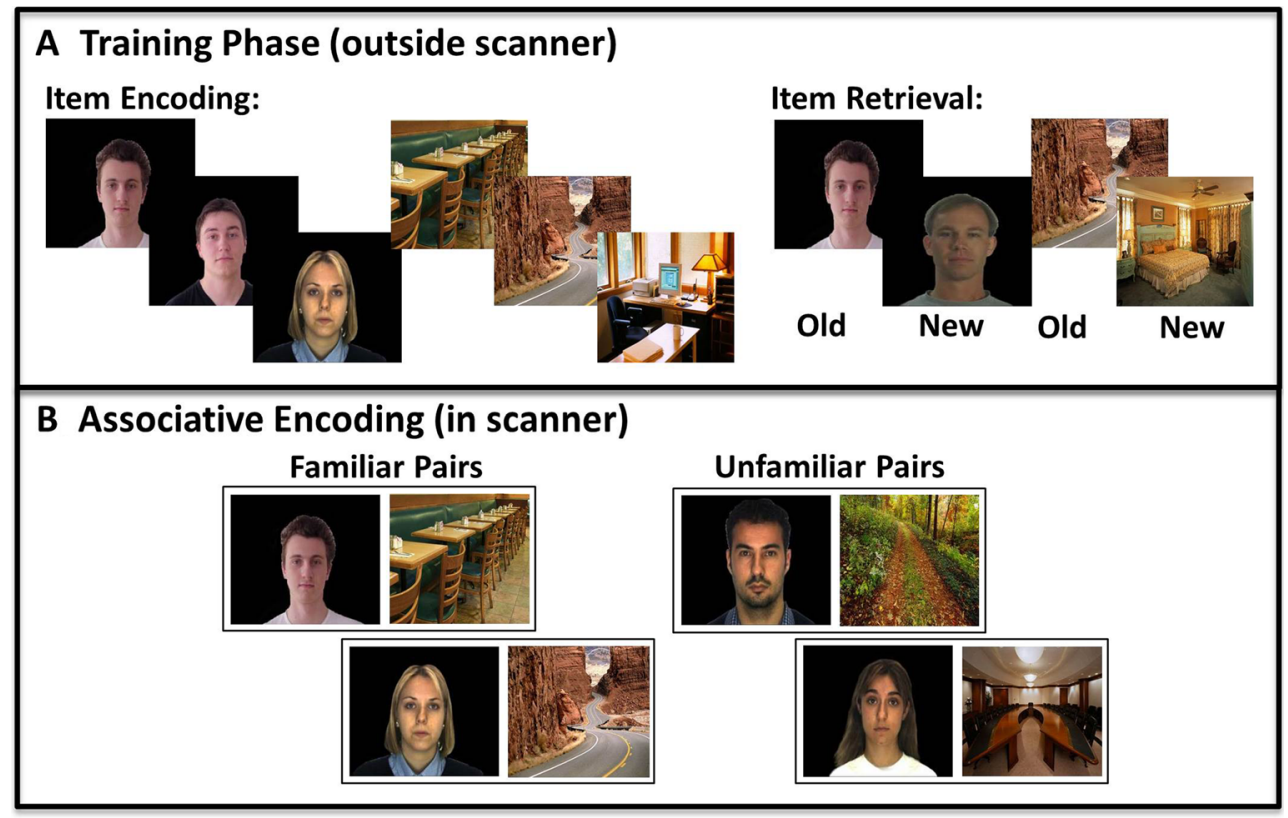

Fig. 1 Task design. (A) During item encoding, participants rated the friendliness of individual faces and the pleasantness of individual scenes. During item retrieval, participants made an old-versus-new memory judgment on the individual faces and scenes. (B) During relational encoding, participants rated how well the face fit with the scene (focusing on the relationship between the two items) for pairs composed of both familiarized-item (trained) and unfamiliarized-item (untrained/novel) pairs. presentation/association. Finally, participants were told to respond "new" if they believed that the exact face-scene pair had not been presented together during the encoding session (even if the individual items had been presented during the encoding phase). It was further made clear that a rating of "New" should be made even if participants remembered having seen a particular face or scene before, but had not seen that specific combination before. Retrieval lasted approximately $25 \mathrm{~min}$ and consisted of five 5-min runs. The retrieval data have been presented in a previous publication (Dennis, Johnson, \& Peterson, 2014).

\section{Image acquisition}

Images were collected using a Siemens 3-T scanner and a 12channel head coil. They were acquired using a T1-weighted sagittal localizer to align the scans to the AC-PC line. Highresolution anatomical images were acquired with a 1,650 -ms repetition time (TR), 2.03-ms echo time (TE), 240-mm field of view (FOV), $256 \times 256$ matrix, 160 axial slices, and $1-\mathrm{mm}$ slice thickness for each participant. Echoplanar functional images were acquired using interleaved acquisition and a 2,500ms TR, 25-ms TE, 240-mm FOV, $80 \times 80$ matrix, and 48 axial slices with a $3.0-\mathrm{mm}$ slice thickness, resulting in $3.0-\mathrm{mm}$ isotropic voxels. The angle of acquisition was set approximately perpendicular to the hippocampus, without sacrificing coverage of the frontal lobes.

\section{Image processing}

Processing of the fMRI data was carried out using SPM8 (Statistical Parametric Mapping; Wellcome Department of Cognitive Neurology, www.fil.ion.ucl.ac.uk/spm). The timeseries data were corrected for differences in slice acquisition times and realigned. Slice time correction and realignment were carried out using the first volume of the first run as the reference slice. With regard to co-registration, the structural images were co-registered to the standardized Montreal Neurological Institute (MNI) space, and information from this step was applied to all functional images during normalization in order to transform the individual images to standard MNI space. No resampling of voxels was conducted. The processed data were then spatially smoothed using an 8-mm isotropic Gaussian kernel.

\section{fMRI analyses}

Trial-related activity was modeled in the general linear model (GLM) with a stick function corresponding to the trial onsets, convolved with a canonical hemodynamic response function. Subsequently remembered trials for the familiarized- and unfamiliarized-item pairs (i.e., encoding trials leading to a "remember" recognition response) were modeled separately. For each trial type, subsequently forgotten trials (i.e., encoding trials leading to a "new" recognition response) and those identified with subsequent familiarity (i.e., encoding trials leading to a "know" recognition response) were combined into a single 
regressor. The foregoing separation of recollection trials from other trial types was carried out for several reasons. First, whereas familiarity represents accurate responding, a recollection response to a face-scene pair indicates the high degree of confidence and specificity of memory detail that we aimed to examine in the present study. Additionally, the average numbers of misses and familiarity responses to targets were relatively low across both conditions (see Table 1 and the Results). Thus, in order to achieve an appropriate level of power necessary in a baseline for comparing recollection effects, we chose to collapse across "know" and "new" responses (for similar analysis approaches, see Dennis et al., 2008; Otten, Quayle, Akram, Ditewig, \& Rugg, 2006; Schon, Hasselmo, Lopresti, Tricarico, $\&$ Stern, 2004). In order to identify activity related to general associative-encoding success, we compared all subsequently remembered trials to the subsequently forgotten trials. Direct comparisons between subsequently remembered familiarized-item pairs and subsequently remembered unfamiliarized-item pairs were conducted to assess differences in the encoding processes mediating associative encoding in the different conditions.

For all contrasts, in order to obtain results that were corrected for multiple comparisons, we used Monte Carlo simulations (https://www2.bc.edu/sd-slotnick/scripts.htm; see Slotnick et al., 2003) to define the individual voxel and cluster extent thresholds. This procedure takes into account the acquisition matrix $(80 \times 80)$, number of slices $(48)$, voxel dimensions $(3 \times 3 \times 3 \mathrm{~mm})$, intrinsic smoothness (13. $3 \mathrm{~mm}$ ), resampling of voxels (none, in the present study), and individual voxel threshold $(p<.01)$ in order to simulate data and estimate the rate of Type I errors associated with a given cluster extent. Using a 10,000-iteration Monte Carlo simulation that incorporated the aforementioned parameters, the simulations suggested that a cluster extent threshold of 18 voxels $\left(486 \mathrm{~mm}^{3}\right)$ should be used to identify results that would be FWE-corrected for multiple comparisons at $p<.05$.

\section{Results}

\section{Behavioral}

Item familiarization task The average hit rates for faces and scenes in the familiarization phase were $.77(S D=.11)$ and .91 $(S D=.09)$, respectively. ${ }^{2}$

Associative memory task Associative hits and false alarms were identified as "remember" responses to intact face-scene pairs and recombined pairs, respectively, at retrieval. Direct comparisons between familiarized-item pairs and

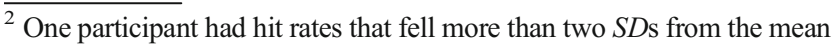
in each category (faces, .44; scenes, .64). An analysis of the imaging data after excluding this individual did not alter the reported results.
}

unfamiliarized-item pairs revealed that participants identified familiarized targets as old at a higher rate than unfamiliarized targets $(M=.65, S D=.19$, vs. $M=.44, S D=.22$, respectively), $t(19)=8.877, p<.001$, and showed higher rates of false alarms to unfamiliarized lures $(M=.19, S D=.12)$ than to familiarized lures $(M=.15, S D=.11), t(19)=-2.218, p=$ .039. Participants also showed higher discriminability (measured by $\left.d^{\prime}\right)$ for familiarized $(M=2.06, S D=1.64)$ than for unfamiliarized $(M=0.82, S D=0.43)$ item pairs, $t(19)=3.601$, $p=.002$. A significant difference was found in "remember" responses between hits and false alarms in both the familiarized, $t(19)=12.27, p<.001$, and unfamiliarized, $t(19)=6.89$, $p<.001$, conditions. The raw "know" responses to hits and false alarms also did not differ from one another in either the familiarized or the unfamiliarized condition $[t(19)=0.198, p=.845$; $t(19)=0.35, p=.727$, respectively] (see Table 1 for a breakdown of the recollection and familiarity response rates). However, when taking into account the assumption that recollection and familiarity are independent processes (Yonelinas \& Jacoby, 1996), the familiarity estimates were $.66(.22)$ for familiarizeditem pairs and .49 (.14) for unfamiliarized-item pairs.

Finally, participants showed significantly reduced rates of false alarms to entirely novel face-scene lures $(M=.012$, $S D=.045)$, relative to both familiarized pairs $(M=0.15, S D=$ $0.11), t(19)=5.92, p<.001$, and unfamiliarized lures $(M=.19$, $S D=.12), t(19)=7.15, p<.001$.

Response time results During the associative-encoding task, participants' speed in making a "goodness-of-fit" rating (see the Method section) to familiarized-item pairs $(M=1,862.34 \mathrm{~ms}$, $S D=152.84)$ was significantly faster than their speed to unfamiliarized-item pairs $(M=1,925.46 \mathrm{~ms}, S D=191.12)$, $t(39)=4.748, p<.001$. A similar pattern was found at retrieval, where participants were also faster to identify familiarized-item pairs as targets $(M=1,679.53 \mathrm{~ms}, S D=219.35)$ than to identify unfamiliarized-item pairs as targets $(M=1,809.57 \mathrm{~ms}, S D=$ 250.23), $t(19)=-3.302, p=.004$ (see Table 1$)$.

\section{fMRI}

Overall, associative encoding elicited activity in the typically observed associative memory network, including bilateral hippocampus and PHG, bilateral fusiform gyrus and occipitotemporal cortex, and the left inferior and middle frontal gyri (see Table 2 for a complete list of regions; see also the Appendix). Activity at encoding was greater for subsequently remembered unfamiliarized-item pairs than for familiarized-item pairs in a large number of brain regions, including the right inferior and superior frontal gyrus, bilateral hippocampus and PHG, and bilateral occipitotemporal cortex, encompassing both the fusiform face area and parahippocampal place area (see Table 3A for a complete list of regions). Conversely, subsequent recollection of familiarized-item pairs exhibited greater 
Table 1 Behavioral results

\begin{tabular}{lll}
\hline & $\begin{array}{l}\text { Familiarized } \\
\text { Mean }(S D)\end{array}$ & $\begin{array}{l}\text { Unfamiliarized } \\
\text { Mean }(S D)\end{array}$ \\
\hline "Remember" Response Rates & & \\
Hits*** & $.65(.19)$ & $.44(.22)$ \\
FA* & $.15(.11)$ & $.19(.12)$ \\
$d^{* *}$ & $2.06(1.64)$ & $0.82(0.43)$ \\
"Know" Response Rates & & \\
Hits & $.24(.16)$ & $.27(.12)$ \\
FA & $.23(.11)$ & $.25(.12)$ \\
$d^{\prime}$ & $0.19(1.29)$ & $0.05(0.48)$ \\
Familiarity Estimates & & $.49(.14)$ \\
Hits*** & $.66(.22)$ & $.32(.14)$ \\
FA & $.28(.15)$ & \\
Response Times (ms) & & $1,925.46(191.12)$ \\
Encoding*** & $1,862.34(152.84)$ \\
Recollection Hits & $1,679.53(219.35)$ & $1,809.57(250.23)$ \\
$\quad$ Retrieval)** & & \\
Familiarity Hits (Retrieval) & $2,133.73(313.88)$ & $2,161.73(347.03)$ \\
\hline
\end{tabular}

The table reports the means and standard deviations of the response rates and response times for familiarized- and unfamiliarized-item pairs. FA = false alarms. Familiarity estimates are calculated as $p_{\text {Know }} \mathrm{Hits} /(1-p$ Remember Hits). ${ }^{*} p<.05,{ }^{* *} p<.005,{ }^{* * *} p<.001$

activity in a relatively limited set of regions, including the left middle frontal gyrus, bilateral inferior parietal cortex, and the precuneus (see Table 3B for a complete list of regions).

In order to investigate whether or not the observed increase in subsequent recollection activity for familiarized-item pairs was related to behavioral benefits, we performed two additional analyses. First, we correlated the mean activity within each parietal cluster with the $d^{\prime}$ scores for familiarized-item pairs. These results revealed a significant correlation between activity differences in the right inferior parietal cortex and performance $(r=.526, p=.01)$. That is, better performance was related to greater differences in parietal activity between familiarized- and unfamiliarized-item pairs. Second, we investigated overlaps in neural activation between the enhancement effects observed for familiarized $>$ unfamiliarized hits and differences in activation between familiarized $>$ unfamiliarized misses. The results showed overlap in both the left inferior parietal cortex and precuneus (but not the right inferior parietal cortex or other regions exhibiting repetition enhancement for familiarized-item pairs). The latter result suggests that whereas increased activity in the left parietal lobe and precuneus likely reflects stimulus repetition, without direct benefits to explicit memory performance, increased activity in the right parietal lobe is related to successful associative memory formation. For completeness of the data, we performed a similar miss analysis for the unfamiliarized $>$ familiarized hit contrast. The results showed that portions, but not all, of the bilateral occipitotemporal activation identified in the hits contrast was also activated in the misses contrast. This suggests that increased activity in bilateral occipitotemporal cortex reflects both general stimulusprocessing effects and successful associative memory formation for unfamiliarized-item pairs, whereas the remaining activation reported in Table $3 \mathrm{~A}$ reflects only successful associative memory formation for unfamiliarized-item pairs.

\section{Discussion}

Past research has indicated that associative memory is a difficult task, especially when compared to other types

Table 2 Associative memory success

\begin{tabular}{|c|c|c|c|c|c|c|c|}
\hline & BA & $\mathrm{H}$ & $\mathrm{X}$ & $\mathrm{Y}$ & $\mathrm{Z}$ & $t$ & $\mathrm{~mm}^{3}$ \\
\hline Orbitofrontal cortex & 10 & M & -9 & 55 & 7 & 3.73 & 2,268 \\
\hline Inferior frontal gyrus & 47 & $\mathrm{~L}$ & -33 & 31 & -10 & 4.23 & 486 \\
\hline Ventromedial PFC & $11 / 25$ & M & 6 & 30 & -15 & 4.14 & 1,674 \\
\hline Middle frontal gyrus & 44 & $\mathrm{~L}$ & -30 & 15 & 26 & 4.04 & 837 \\
\hline Hippocampus/Parahippocampal gyrus & & $\mathrm{R}$ & 27 & -19 & -10 & 3.67 & 1,620 \\
\hline Hippocampus/Parahippocampal gyrus & & $\mathrm{L}$ & -32 & -31 & -9 & 3.72 & 1,620 \\
\hline Fusiform gyrus & $36 / 37$ & $\mathrm{R}$ & 36 & -39 & -9 & 4.07 & 891 \\
\hline Fusiform gyrus & $36 / 37$ & $\mathrm{~L}$ & -36 & -34 & -12 & 4.77 & 1,890 \\
\hline Middle temporal gyrus & 21 & $\mathrm{R}$ & 62 & -4 & -11 & 3.76 & 1,296 \\
\hline Temporal pole & 38 & $\mathrm{~L}$ & -39 & 13 & -22 & 3.00 & 1,431 \\
\hline Occipitoparietal cortex & 39 & $\mathrm{~L}$ & -45 & -72 & 28 & 3.45 & 2,592 \\
\hline Occipitoparietal cortex & 39 & $\mathrm{R}$ & 50 & -69 & 33 & 4.06 & 1,674 \\
\hline
\end{tabular}

$\mathrm{BA}=$ Brodmann's area; $\mathrm{H}=$ hemisphere $\mathrm{L}=$ left $\mathrm{R}=$ right $\mathrm{M}=$ medial $t=$ statistical $t$ value $; \mathrm{mm}^{3}=$ millimeters cubed. Associative memory success is defined as all recollected ("Remember") item pairs as compared to both familiar ("Know") pairs and misses ("New") 
Table 3 Difference in associative encoding success as a function of item familiarity

\begin{tabular}{|c|c|c|c|c|c|c|c|}
\hline & BA & $\mathrm{H}$ & $X$ & $Y$ & $Z$ & $t$ & $\mathrm{~mm}^{3}$ \\
\hline \multicolumn{8}{|c|}{ A. Unfamiliarized $>$ Familiarized } \\
\hline \multirow[t]{2}{*}{ Inferior frontal gyrus } & $45 / 46$ & $\mathrm{R}$ & 39 & 29 & 14 & 5.95 & 2,619 \\
\hline & $9 / 44$ & $\mathrm{R}$ & 42 & 15 & 26 & 4.34 & 2,187 \\
\hline Superior frontal gyrus & 9 & $\mathrm{R}$ & 27 & 53 & 30 & 3.37 & 486 \\
\hline \multirow[t]{2}{*}{ Precentral gyrus } & 6 & $\mathrm{R}$ & 48 & -9 & 11 & 3.34 & 594 \\
\hline & 4 & $\mathrm{R}$ & 50 & -5 & 30 & 2.98 & 513 \\
\hline Postcentral gyrus & 3 & $\mathrm{R}$ & 50 & -17 & 28 & 3.38 & 702 \\
\hline Putamen & & $\mathrm{R}$ & 9 & 7 & -11 & 4.01 & 594 \\
\hline \multirow[t]{2}{*}{ Hippocampus/PHG } & & $\mathrm{R}$ & 30 & -36 & -9 & 4.36 & 891 \\
\hline & & $\mathrm{L}$ & -30 & -39 & -6 & 4.8 & 1,215 \\
\hline \multirow[t]{3}{*}{ Superior temporal gyrus } & $22 / 42$ & $\mathrm{R}$ & 56 & -41 & 12 & 3.69 & 2,322 \\
\hline & 42 & $\mathrm{~L}$ & -48 & -32 & 15 & 3.63 & 648 \\
\hline & 38 & $\mathrm{~L}$ & -50 & -4 & -8 & 3.11 & 486 \\
\hline \multirow[t]{2}{*}{ Occipitotemporal cortex } & $19 / 37$ & $\mathrm{~L}$ & -36 & -82 & 17 & 7.13 & 21,519 \\
\hline & $19 / 37$ & $\mathrm{R}$ & 42 & -73 & 19 & 7.45 & 42,498 \\
\hline Cerebellum & - & $\mathrm{L}$ & -12 & -75 & -27 & 4.66 & 3,753 \\
\hline \multicolumn{8}{|c|}{ B. Familiarized $>$ Unfamiliarized } \\
\hline \multirow[t]{2}{*}{ Inferior parietal lobe } & $39 / 40$ & $\mathrm{R}^{\wedge}$ & 53 & -60 & 35 & 5.32 & 3,753 \\
\hline & $39 / 40$ & $\mathrm{~L}^{*}$ & -45 & -61 & 33 & 4.97 & 6,183 \\
\hline Precuneus & $7 / 31$ & $\mathrm{M}^{*}$ & 3 & -58 & 30 & 3.87 & 5,238 \\
\hline Middle temporal gyrus & 21 & $\mathrm{~L}$ & -53 & -31 & -9 & 3.48 & 594 \\
\hline Middle frontal gyrus & 8 & $\mathrm{~L}$ & -39 & 22 & 42 & 3.28 & 594 \\
\hline Middle frontal gyrus & $46 / 9$ & $\mathrm{~L}$ & -39 & 27 & 28 & 2.91 & 540 \\
\hline
\end{tabular}

$\mathrm{BA}=$ Brodmann's area; $\mathrm{H}=$ hemisphere; $\mathrm{L}=$ left; $\mathrm{R}=$ right; $\mathrm{M}=$ medial; $t=$ statistical $t$ value; $\mathrm{mm}^{3}=$ millimeters cubed. ${ }^{*}$ Activity overlapped with familiarized $>$ unfamiliarized misses. ^Activity corrected with familiarized $d^{\prime}$ scores

of memory, such as item memory (Castel \& Craik, 2003; Gold et al., 2006; Naveh-Benjamin, 2000; Tulving \& Schacter, 1990; Yonelinas, 1997; Yonelinas et al., 2007). One theoretical account for this difference includes the assumption that an increased workload is imposed by the need to simultaneously learn both item and associative information (Chalfonte \& Johnson, 1996; Naveh-Benjamin, 2000; Overman \& Becker, 2009). In the present study, we sought to test this account by identifying differences in neural activation that were dependent on whether individuals were required to concurrently learn item information alongside associative information. Overall, our results showed that successful associative encoding was supported by a network of brain regions previously shown to support the binding of information, including bilateral hippocampus, visual cortices, and PFC (Dennis et al., 2008; Giovanello \& Schacter, 2012; Giovanello et al., 2004; Slotnick et al., 2003; Yonelinas et al., 2001). However, when participants' previous experience with the associative items was taken into account, neural differences emerged.
As was evidenced by the higher accuracy and faster response times to familiarized-item than to unfamiliarizeditem pairs, exposing participants to the individual items prior to associative encoding facilitated subsequent recollection, which is suggestive of a reduction of difficulty in the familiarized condition. Additionally, increased familiarity with items resulted in reduced activity across a large number of brain regions during associative encoding, including the fusiform face area, parahippocampal place area, hippocampus, and superior frontal gyrus (see Table 3A). This aligns with previous encoding studies that have observed repetition suppression effects within a similar set of brain regions when the encoding stimuli are repeated across multiple trials (Duzel et al., 2003; Kremers et al., 2014; Vannini et al., 2013). Such reductions in activation have been associated with increased efficiency in processing items that have been previously presented (cf. priming; Badgaiyan \& Posner, 1997; Desimone, 1996; Rugg, Soardi, \& Doyle, 1995; Wiggs \& Martin, 1994). Although prior neuroimaging studies have investigated repetition effects associated with the repetition of item-item pairs (Duzel et al., 2003; Sperling et al., 2003), no prior study had 
examined the benefit of individual item familiarization on the subsequent associative encoding of item pairs. Our study extends prior work in the area of associative memory by showing reductions in neural processing within the foregoing brain regions when pairs were novel and only the individual items had been previously studied. Specifically, we found that despite the novel face-scene pair configuration in both encoding conditions, the familiarized condition exhibited reduced activation in regions known for processing both faces and scenes (i.e., the fusiform face area and parahippocampal place area), as compared to the unfamiliarized condition (see Fig. 2A). Thus, our results suggest that repetition suppression effects in the abovementioned brain regions represent a sharpening of the neural responses to specific stimuli (i.e., individual items) that can, in turn, facilitate later associative memory processing, leading to enhanced performance.

The familiarized condition also resulted in reduced activity in regions known to support item-item binding, including the hippocampus and superior frontal gyrus (Davachi, Mitchell, \& Wagner, 2003; Jackson \& Schacter, 2004; Mayes et al., 2004; Ranganath, Cohen, Dam, \& D'Esposito, 2004). Although previous item- and associative-encoding studies had shown repetition suppression in the MTL (Kremers et al., 2014; Vannini et al., 2013), it was unclear whether the same finding would emerge in the present study, given that all of the associations were novel during encoding. We had predicted that although prior familiarization of items would reduce the need to encode item-specific features at the time of associative encoding, the needs to bind item-item information would still be relatively similar across conditions. Thus, we did not predict neural differences within regions supporting binding, particularly the hippocampus. However, the present results suggest that during associative encoding, the processing advantage created by prior familiarization with items extends to hippocampal binding, as well. Specifically, our results suggest that hippocampal recruitment in support of successful associative encoding is reduced when only the associative link between items must be formed, as opposed to when the encoding of both item and associative information is required. This has been the first neuroimaging study to demonstrate a reduction in associative processing load as a result of prior experience with to-be-associated items.

In addition to the observed reductions in neural activity, familiarization of items prior to associative encoding resulted in increased activity within the parietal cortex, including bilateral inferior parietal cortex and precuneus, relative to the unfamiliarized condition (see Fig. 2B). The parietal cortex is associated with goal-directed attention (see Corbetta, Patel, \& Shulman, 2008; Corbetta \& Shulman, 2002; Wager \& Smith, 2003) and the integration of information into a unified representation (e.g., Fitzgerald, Freedman, \& Assad, 2011; Freedman \& Assad, 2006; Fuster \& Bressler, 2012). Therefore, the repetition enhancement effects in parietal cortices observed during associative encoding have been interpreted as reflecting the formation of new associations that help build complex memories (Fitzgerald et al., 2011; Kremers et al., 2014; Vannini et al., 2013). The present study extends these findings by showing enhancement effects in parietal cortex when the items related in the associations have been previously studied but the associations themselves have not. That is, our results suggest that the neural processes underlying the formation of novel associations between items are facilitated when the items have an existing representation in memory, as compared to when the items are themselves novel.

To further investigate the role of repetition enhancement with respect to successful associative encoding, we performed two additional analyses. We first correlated the mean activity within

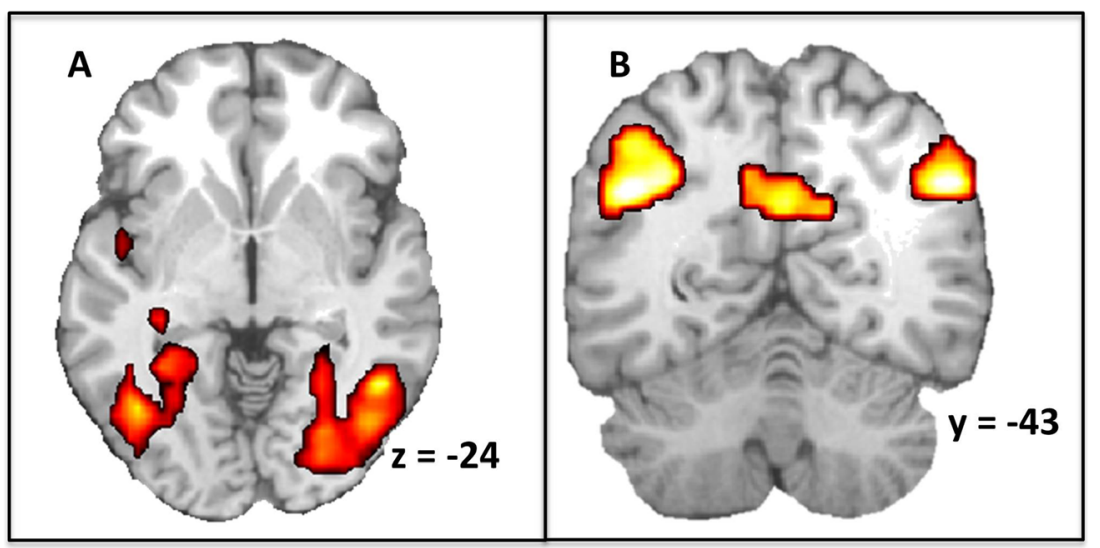

Fig. 2 Neural differences between trial types. (A) Greater activity in occipitotemporal regions, including bilateral fusiform face area and parahippocampal place area, supporting unfamiliarized $>$ familiarized associative encoding. (B) Greater activity in bilateral inferior parietal cortex and precuneus supporting familiarized $>$ unfamiliarized associative encoding. Both the left parietal and precuneus clusters overlapped with regions identified in the familiarized $>$ unfamiliarized miss analysis, whereas the right parietal cluster activation significantly correlated with $d^{\prime}$ scores for familiarized-item pairs. 
each cluster with $d^{\prime}$ scores for familiarized-item pairs, and second, we investigated the overlap between the aforementioned enhancement effects and any differences in activation between familiarized and unfamiliarized misses. Whereas the former effects would lend support to our conclusion that this represents successful processing, the latter would be suggestive of item repetition effects that aided processing, but not success. Our results revealed a significant correlation between repetition enhancement effects in the right inferior parietal cortex and encoding success. No correlation with behavior was observed for either the left parietal cluster or the precuneus. In addition, both the left parietal and precuneus clusters partially overlapped with areas identified in the miss analysis. Taken together, these results suggest that a dissociation may exist between left and right inferior parietal cortex with respect to the roles that they play in associative encoding. Whereas the left region may mediate attentional processes supporting facilitated processing of associative information following the repetition of items, the right may mediate successful associative binding of familiarized-item pairs. Additional work will be needed to investigate this observation.

In sum, the repetition suppression results described above and the repetition enhancement results in the parietal cortex suggest that prior familiarization with information reduces the need to encode item identity during associative encoding, allowing for a shift in neural resources to regions that support associative processing. Behavioral performance differences between the two associative-encoding conditions also support the explanation that prior familiarity with items reduces the need to divide resources between item and associative encoding. A higher hit rate and $d^{\prime}$, as well as reduced response times, in the familiarized-item pair condition suggests that more resources were available to successfully encode the relationship between items when the items themselves were previously known to the participant. This opens potential avenues for the enhancement of associative memory performance through prior exposure of the to-be-associated pieces of information, without requiring specific training on the pairs as a whole.

These results have implications for improving performance in groups, such as the elderly, who exhibit deficits in associative memory tasks. Although prior behavioral work has shown that repetition of pairs improves older adults' associative memory performance, the contribution of item familiarity to associative memory has been less clear (Kilb \& NavehBenjamin, 2011). By examining the neural underpinnings of item and pair repetition in associative memory, it may be possible to identify how the brain regions involved in item and pair familiarity support associative processes, which may allow more precisely targeted interventions to improve memory performance. For example, prior behavioral studies have demonstrated that age-related associative memory deficits are linked to the increased task complexity of associative memory over that of item memory, as well as to age-related reductions in processing resources (Chalfonte \& Johnson, 1996; NavehBenjamin, 2000; Overman \& Becker, 2009; Overman \& Stephens, 2013). The present study has demonstrated reduced neural activity in several brain regions that have been shown to support associative encoding. This suggests that if the demands incurred by neural processing during associative memory tasks can be lessened by prior exposure to the individual items, more resources would then be made available to older adults for processing the associative information (for behavioral work, see Naveh-Benjamin, 2000). Future work will be needed to investigate this mechanism in older adults.

\section{Conclusions}

The need to concurrently process both item and associative information during encoding tasks may place an undue burden on the associative-encoding network. Prior behavioral work has suggested that this burden may be reduced if solely associative information is encoded. To this end, in the present study we investigated the effect of item familiarity in associative memory, by directly contrasting the neural activity supporting successful associative encoding under two conditions: one in which items were previously studied but the associative information was novel, and a second in which both the items and associations were novel and needed to be learned concurrently. The study yielded two main findings. First, prior familiarity with items resulted in reduced activity across a large number of brain regions, including those associated with item-specific processing (i.e., the fusiform face area and parahippocampal place area) and item-item binding (hippocampus). Second, increased activity following familiarization was localized to a small set of regions including bilateral parietal cortex, known for mediating associative processing. Whereas the left parietal lobe and precuneus appear to mediate attentional processes that support the processing of associative information following repetition of items, our results suggest that the right inferior parietal cortex mediated successful associative binding of familiarized-item pairs. Taken together, these results suggest that prior experience with items influences how they are processed during associative encoding, and that this prior exposure can lessen the demands incurred by neural processing in an associative encoding task. This can, in turn, focus neural resources within regions supporting the formation of associative links, thereby leading to enhanced associative memory performance.

Author note The authors thank Kristina Peterson for assistance in the data collection and analysis, and the Penn State Social, Life, \& Engineering Sciences Imaging Center (SLEIC), 3T MRI Facility. This work was supported by a National Science Foundation (NSF) grant (BCS1025709) awarded to N.A.D. and by a Graduate Research Fellowship from the NSF awarded to I.C.T. (DGE1255832). Any opinions, findings, and 
conclusions or recommendations expressed in this material are those of the authors and do not necessarily reflect the views of the NSF. This research was conducted while N.A.D. was a Research Grant recipient from the American Federation for Aging
Research. Portions of the research in this article used the Color FERET database of facial images collected under the FERET program, sponsored by the Department of Defense Counterdrug Technology Development Program Office.

\section{Appendix}

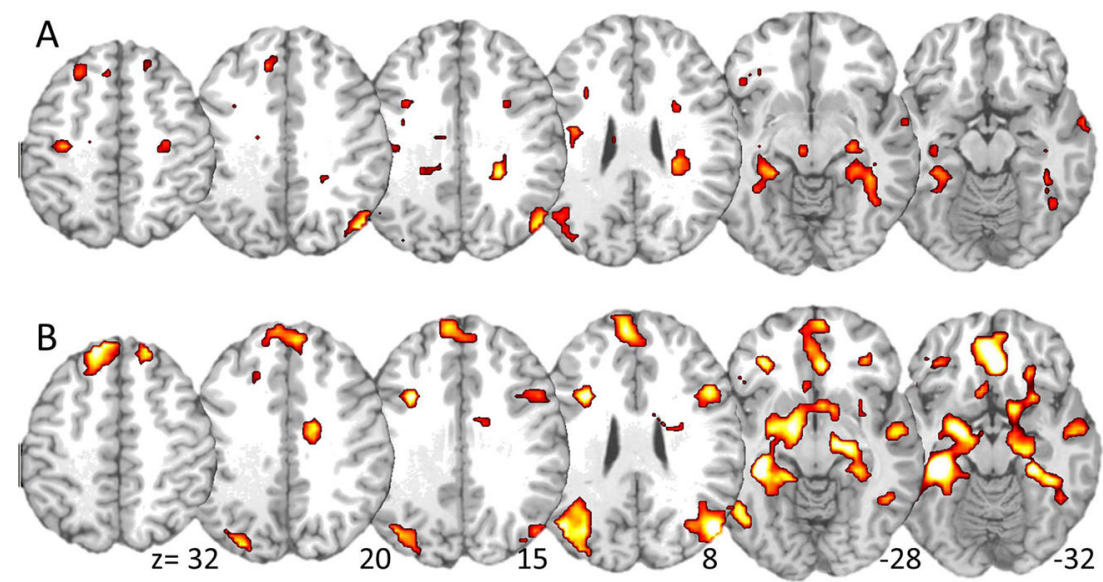

Fig. A1 Subsequent recollection activity in the (A) familiarized-item and (B) unfamiliarized-item conditions

\section{References}

Badgaiyan, R. D., \& Posner, M. I. (1997). Time course of cortical activations in implicit and explicit recall. Journal of Neuroscience, 17, 4904-4913.

Brewer, J. B., Zhao, Z., Desmond, J. E., Glover, G. H., \& Gabrieli, J. D. E. (1998). Making memories: Brain activity that predicts how well visual experience will be remembered. Science, 281, 1185-1187. doi:10.1126/science. 281.5380 .1185

Castel, A. D., \& Craik, F. I. M. (2003). The effects of aging and divided attention on memory for item and associative information. Psychology and Aging, 18, 873-885.

Chalfonte, B. L., \& Johnson, M. K. (1996). Feature memory and binding in young and older adults. Memory \& Cognition, 24, 403-416. doi: 10.3758/BF03200930

Corbetta, M., Patel, G., \& Shulman, G. L. (2008). The reorienting system of the human brain: From environment to theory of mind. Neuron, 58, 306-324. doi:10.1016/j.neuron.2008.04.017

Corbetta, M., \& Shulman, G. L. (2002). Control of goal-directed and stimulus-driven attention in the brain. Nature Reviews Neuroscience, 3, 201-215.

Davachi, L. (2006). Item, context and relational episodic encoding in humans. Current Opinion in Neurobiology, 16, 693-700.

Davachi, L., Mitchell, J. P., \& Wagner, A. D. (2003). Multiple routes to memory: Distinct medial temporal lobe processes build item and source memories. Proceedings of the National Academy of Sciences, 100, 2157-2162.

Dennis, N. A., Hayes, S. M., Prince, S. E., Madden, D. J., Huettel, S. A., \& Cabeza, R. (2008). Effects of aging on the neural correlates of successful item and source memory encoding. Journal of Experimental Psychology: Learning, Memory, and Cognition, 34, 791-808. doi:10.1037/0278-7393.34.4.791
Dennis, N. A., Johnson, C. E., \& Peterson, K. M. (2014). Neural correlates underlying true and false associative memories. Brain and Cognition, 88, 65-72. doi:10.1016/j.bandc.2014.04.009

Desimone, R. (1996). Neural mechanisms for visual memory and their role in attention. Proceedings of the National Academy of Sciences, 93, 13494-13499.

Diana, R. A., Yonelinas, A. P., \& Ranganath, C. (2007). Imaging recollection and familiarity in the medial temporal lobe: A threecomponent model. Trends in Cognitive Sciences, 11, 379-386. doi: 10.1016/j.tics.2007.08.001

Dolan, R. J., \& Fletcher, P. C. (1997). Dissociating prefrontal and hippocampal function in episodic memory encoding. Nature, 388, 582585.

Duzel, E., Habib, R., Rotte, M., Guderian, S., Tulving, E., \& Heinze, H. J. (2003). Human hippocampal and parahippocampal activity during visual associative recognition memory for spatial and nonspatial stimulus configurations. Journal of Neuroscience, 23, 9439-9444.

Earles, J. L., \& Kersten, A. W. (2008). Effects of age and repetition on the binding of actors and actions. Poster presented at the Cognitive Aging Conference, Atlanta, GA.

Fitzgerald, J. K., Freedman, D. J., \& Assad, J. A. (2011). Generalized associative representations in parietal cortex. Nature Neuroscience, 14. 1075-1079. doi:10.1038/nn.2878

Freedman, D. J., \& Assad, J. A. (2006). Experience-dependent representation of visual categories in parietal cortex. Nature, 443, 85-88. doi: 10.1038/nature05078

Fuster, J. M., \& Bressler, S. L. (2012). Cognit activation: A mechanism enabling temporal integration in working memory. Trends in Cognitive Sciences, 16, 207-218. doi:10.1016/j.tics.2012.03.005

Giovanello, K. S., \& Schacter, D. L. (2012). Reduced specificity of hippocampal and posterior ventrolateral prefrontal activity during relational retrieval in normal aging. Journal of Cognitive Neuroscience, 24, 159-170. doi:10.1162/jocn_a_00113 
Giovanello, K. S., Schnyer, D. M., \& Verfaellie, M. (2004). A critical role for the anterior hippocampus in relational memory: Evidence from an fMRI study comparing associative and item recognition. Hippocampus, 14, 5-8.

Gold, J. J., Hopkins, R. O., \& Squire, L. R. (2006). Single-item memory, associative memory, and the human hippocampus. Learning and Memory, 13, 644-649. doi:10.1101/LM.258406

Gonsalves, B. D., Kahn, I., Curran, T., Norman, K. A., \& Wagner, A. D. (2005). Memory strength and repetition suppression: Multimodal imaging of medial temporal cortical contributions to recognition. Neuron, 47, 751-761.

Graf, P., \& Schacter, D. L. (1987). Selective effects of interference on implicit and explicit memory for new associations. Journal of Experimental Psychology: Learning, Memory, and Cognition, 13, 45-53. doi:10.1037/0278-7393.13.1.45

Gruber, T., \& Muller, M. M. (2002). Effects of picture repetition on induced gamma band responses, evoked potentials, and phase synchrony in the human EEG. Cognitive Brain Research, 13, 377-392. doi:10.1016/S0926-6410(01)00130-6

Gruber, T., \& Muller, M. M. (2005). Oscillatory brain activity dissociates between associative stimulus content in a repetition priming task in the human EEG. Cerebral Cortex, 15, 109-116. doi:10.1093/cercor/ bhh 113

Hockley, W. E., \& Consoli, A. (1999). Familiarity and recollection in item and associative recognition. Memory \& Cognition, 27, 657-664. doi:10.3758/BF03211559

Jackson, O., 3rd, \& Schacter, D. L. (2004). Encoding activity in anterior medial temporal lobe supports subsequent associative recognition. NeuroImage, 21, 456-462.

Kanwisher, N., McDermott, J., \& Chun, M. M. (1997). The fusiform face area: A module in human extrastriate cortex specialized for face perception. Journal of Neuroscience, 17, 4302-4311.

Kilb, A., \& Naveh-Benjamin, M. (2007). Paying attention to binding: Further studies assessing the role of reduced attentional resources in the associative deficit of older adults. Memory \& Cognition, 35, 1162-1174. doi:10.3758/BF03193486

Kilb, A., \& Naveh-Benjamin, M. (2011). The effects of pure pair repetition on younger and older adults' associative memory. Journal of Experimental Psychology: Learning, Memory, and Cognition, 37, 706-719. doi:10.1037/A0022525

Kremers, N. A. W., Deuker, L., Kranz, T. A., Oehrn, C., Fell, J., \& Axmacher, N. (2014). Hippocampal control of repetition effects for associative stimuli. Hippocampus, 24, 892-902. doi:10.1002/ hipo. 22278

Light, L. L., Patterson, M. M., Chung, C., \& Healy, M. R. (2004). Effects of repetition and response deadline on associative recognition in young and older adults. Memory \& Cognition, 32, 1182-1193. doi:10.3758/BF03196891

Martinez, A. M., \& Benavente, R. (1998). The AR face database (CVC Technical Report No. 24). Retrieved from http://www2.ece.ohiostate.edu/ aleix/ARdatabase.html

Mayes, A. R., Holdstock, J. S., Isaac, C. L., Montaldi, D., Grigor, J., Gummer, A., . . . Norman, K. A. (2004). Associative recognition in a patient with selective hippocampal lesions and relatively normal item recognition. Hippocampus, 14, 763-784.

Minear, M., \& Park, D. C. (2004). A lifespan database of adult facial stimuli. Behavior Research Methods, Instruments, \& Computers, 36, 630-633. doi:10.3758/BF03206543

Naveh-Benjamin, M. (2000). Adult age differences in memory performance: Tests of an associative deficit hypothesis. Journal of Experimental Psychology: Learning, Memory, and Cognition, 26, 1170-1187. doi:10.1037/0278-7393.26.5.1170

Otten, L. J., Quayle, A. H., Akram, S., Ditewig, T. A., \& Rugg, M. D. (2006). Brain activity before an event predicts later recollection. Nature Neuroscience, 9, 489-491. doi:10.1038/nn1663
Overman, A. A., \& Becker, J. T. (2009). The associative deficit in older adult memory: Recognition of pairs is not improved by repetition. Psychology and Aging, 24, 501-506. doi:10.1037/A0015086

Overman, A. A., \& Stephens, J. D. W. (2013). Synergistic effects of encoding strategy and context salience on associative memory in older adults. Psychology and Aging, 28, 654-665. doi:10.1037/ A0031441

Phillips, P. J., Moon, H., Rizvi, S. A., \& Rauss, P. J. (2000). The FERET evaluation methodology for face-recognition algorithms. IEEE Transactions on Pattern Analysis and Machine Intelligence, 22, 1090-1104. doi:10.1109/34.879790

Phillips, P. J., Wechsler, H., Huang, J., \& Rauss, P. J. (1998). The FERET database and evaluation procedure for face-recognition algorithms. Image and Vision Computing, 16, 295-306. doi:10.1016/S02628856(97)00070-X

Prince, S. E., Dennis, N. A., \& Cabeza, R. (2009). Encoding and retrieving faces and places: Distinguishing process- and stimulus-specific differences in brain activity. Neuropsychologia, 47, 2282-2289.

Puce, A., Allison, T., Gore, J. C., \& McCarthy, G. (1995). Face-sensitive regions in human extrastriate cortex studied by functional MRI. Journal of Neurophysiology, 74, 1192-1199.

Rand-Giovannetti, E., Chua, E. F., Driscoll, A. E., Schacter, D. L., Albert, M. S., \& Sperling, R. A. (2006). Hippocampal and neocortical activation during repetitive encoding in older persons. Neurobiology of Aging, 27, 173-182. doi:10.1016/j.neurobiolaging.2004.12.013

Ranganath, C. (2010a). Binding items and contexts: The cognitive neuroscience of episodic memory. Current Directions in Psychological Science, 19, 131-137. doi:10.1177/0963721410368805

Ranganath, C. (2010b). A unified framework for the functional organization of the medial temporal lobes and the phenomenology of episodic memory. Hippocampus, 20, 1263-1290. doi:10.1002/hipo.20852

Ranganath, C., Cohen, M. X., Dam, C., \& D’Esposito, M. (2004). Inferior temporal, prefrontal, and hippocampal contributions to visual working memory maintenance and associative memory retrieval. Journal of Neuroscience, 24, 3917-3925. doi:10.1523/ JNEUROSCI.5053-03.2004

Rossion, B., Schiltz, C., \& Crommelinck, M. (2003). The functionally defined right occipital and fusiform "face areas" discriminate novel from visually familiar faces. NeuroImage, 19, 877-883.

Rugg, M. D., Soardi, M., \& Doyle, M. C. (1995). Modulation of eventrelated potentials by the repetition of drawings of novel objects. Cognitive Brain Research, 3, 17-24.

Schon, K., Hasselmo, M. E., Lopresti, M. L., Tricarico, M. D., \& Stern, C. E. (2004). Persistence of parahippocampal representation in the absence of stimulus input enhances long-term encoding: A functional magnetic resonance imaging study of subsequent memory after a delayed match-to-sample task. Journal of Neuroscience, 24, 11088-11097. doi:10.1523/JNEUROSCI.3807-04.2004

Slotnick, S. D., Moo, L. R., Segal, J. B., \& Hart, J., Jr. (2003). Distinct prefrontal cortex activity associated with item memory and source memory for visual shapes. Cognitive Brain Research, 17, 75-82. doi:10.1016/S0926-6410(03)00082-X

Solina, F., Peer, P., Batageli, B., Juvan, S., \& Kovac, J. (2003, March). Color-based face detection in the "15 seconds of fame" art installation. Paper presented at the Conference on Computer Vision/Computer Graphics Collaboration for Model-based Imaging, Rendering, Image Analysis and Graphical Special Effects, INRIA Rocquencourt, France.

Sperling, R. A., Bates, J. F., Chua, E. F., Cocchiarella, A. J., Rentz, D. M., Rosen, B. R., . . Albert, M. S. (2003). fMRI studies of associative encoding in young and elderly controls and mild Alzheimer's disease. Journal of Neurology, Neurosurgery, and Psychiatry, 74, 44 50 .

Staresina, B. P., \& Davachi, L. (2008). Selective and shared contributions of the hippocampus and perirhinal cortex to episodic item and 
associative encoding. Journal of Cognitive Neuroscience, 20, 14781489. doi:10.1162/jocn.2008.20104

Tulving, E., \& Schacter, D. L. (1990). Priming and human memory systems. Science, 247, 301-306. doi:10.1126/science.2296719

Vannini, P., Hedden, T., Sullivan, C., \& Sperling, R. A. (2013). Differential functional response in the posteromedial cortices and hippocampus to stimulus repetition during successful memory encoding. Human Brain Mapping, 34, 1568-1578. doi:10.1002/hbm.22011

Wager, T. D., \& Smith, E. E. (2003). Neuroimaging studies of working memory: A meta-analysis. Cognitive, Affective, \& Behavioral Neuroscience, 3, 255-274. doi:10.3758/CABN.3.4.255

Wiggs, C. L., \& Martin, A. (1994). Aging and feature-specific priming of familiar and novel stimuli. Psychology and Aging, 9, 578-588.

Wiggs, C. L., \& Martin, A. (1998). Properties and mechanisms of perceptual priming. Current Opinion in Neurobiology, 8, 227-233.
Yonelinas, A. P. (1997). Recognition memory ROCs for item and associative information: The contribution of recollection and familiarity. Memory \& Cognition, 25, 747-763. doi:10.3758/ BF03211318

Yonelinas, A. P., Hopfinger, J. B., Buonocore, M. H., Kroll, N. E., \& Baynes, K. (2001). Hippocampal, parahippocampal and occipitaltemporal contributions to associative and item recognition memory: An fMRI study. NeuroReport, 12, 359-363.

Yonelinas, A. P., \& Jacoby, L. L. (1996). Noncriterial recollection: Familiarity as automatic, irrelevant recollection. Consciousness and Cognition, 5, 131-141. doi:10.1006/ccog.1996.0008

Yonelinas, A. P., Widaman, K., Mungas, D., Reed, B., Weiner, M. W., \& Chui, H. C. (2007). Memory in the aging brain: Doubly dissociating the contribution of the hippocampus and entorhinal cortex. Hippocampus, 17, 1134-1140. 